\title{
Highly Efficient Access to Strained Bicyclic Ketals via Gold-catalyzed Cycloisomerization of Bis-homopropargylic Diols
}

\author{
Sylvain Antoniotti, Emilie Genin, Véronique Michelet,* and Jean-Pierre Genêt*
}

\section{Supporting information}

$\mathrm{AuCl}$ and $\mathrm{AuCl}_{3}$ were purchased from Cortecnet. All manipulations were carried out under argon and Schlenk techniques for catalytic tests. Solvents were degassed by sparging with argon and/or exposure to vacuum. ${ }^{1} \mathrm{H}$ NMR and ${ }^{13} \mathrm{C}$ NMR were recorded on a Bruker AV 300 instrument. All signals were expressed as ppm down field from $\mathrm{Me}_{4} \mathrm{Si}$ for ${ }^{1} \mathrm{H}$ and ${ }^{13} \mathrm{C} \mathrm{NMR}$ used as an internal standard $(\delta)$. Coupling constants $(J)$ are reported in $\mathrm{Hz}$ and refer to apparent peak multiplicities. Mass spectrometry analyses (direct introduction by chemical ionization with ammoniac) were performed at the Ecole Nationale Supérieure de Chimie de Paris. High resolution mass spectra were performed on a Varian MAT311 instrument at the Ecole Normale Supérieure (Paris).

Standard procedure: a mixture of bis-homopropargylic diol, $\mathrm{AuCl}$ or $\mathrm{AuCl}_{3}(2 \mathrm{~mol} \%)$ in degassed methanol (0.5 molL-1) was stirred under argon atmosphere at room temperature. After completion of the reaction, the mixture was filtered through a short pad of celite (EtOAc) and the solvents were evaporated under reduced pressure to give the corresponding ketal.

1-Methyl-4-[3'-phenylprop-2'-enyl]-2,6-dioxabicyclo[2.2.1]heptane 2a

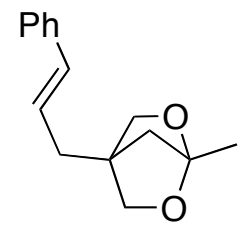

${ }^{1} \mathrm{H}-\mathrm{NMR}\left(300 \mathrm{MHz}, \mathrm{CDCl}_{3}\right): \delta 1.55(\mathrm{~s}, 3 \mathrm{H}), 1.77(\mathrm{~s}, 2 \mathrm{H}), 2.50(\mathrm{~d}, J=7,53 \mathrm{~Hz}, 2 \mathrm{H})$, $3.82(\mathrm{~m}, 4 \mathrm{H}), 6.13(\mathrm{td}, J=7,53 \mathrm{~Hz}, J=15,66 \mathrm{~Hz}, 1 \mathrm{H}), 6,43(\mathrm{~d}, J=15,66 \mathrm{~Hz}, 1 \mathrm{H}), 7.13-$ $7.27(\mathrm{~m}, 5 \mathrm{H}) .{ }^{13} \mathrm{C}-\mathrm{NMR}\left(75 \mathrm{MHz}, \mathrm{CDCl}_{3}\right): \delta 17.9,32.8,44.2,48.7,75.8,108.4$, 117.8, 133.3. CI-MS $\left(\mathrm{NH}_{3}\right): \mathrm{m} / \mathrm{z} 231[\mathrm{M}+\mathrm{H}]^{+}$. DCI/ $\mathrm{NH}_{3}-\mathrm{HRMS}$ Calculated for $\mathrm{C}_{15} \mathrm{H}_{19} \mathrm{O}_{2}: 231.1385$. Found: 231.1388 .

4-Benzyl-1-methyl-2,6-dioxabicyclo[2.2.1]heptane $2 \mathbf{b}$

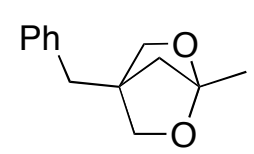

${ }^{1} \mathrm{H}-\mathrm{NMR}\left(300 \mathrm{MHz}, \mathrm{CDCl}_{3}\right): \delta 1.51(\mathrm{~s}, 3 \mathrm{H}), 1.70(\mathrm{~s}, 2 \mathrm{H}), 2.87(\mathrm{~s}, 2 \mathrm{H}), 3.74(\mathrm{~m}, 4 \mathrm{H})$, 7.00-7.40 (m, 5H). ${ }^{13} \mathrm{C}-\mathrm{NMR}\left(75 \mathrm{MHz}, \mathrm{CDCl}_{3}\right): \delta 17.9,35.2,44.6,49.9,76.2,108.3$, 126.7, 128.5, 129.1, 137.3. CI-MS $\left(\mathrm{NH}_{3}\right): \mathrm{m} / \mathrm{z} 222\left[\mathrm{M}+\mathrm{NH}_{4}\right]^{+}, 205[\mathrm{M}+\mathrm{H}]^{+}$. DCI/ $\mathrm{NH}_{3}$-HRMS Calcd for $\mathrm{C}_{13} \mathrm{H}_{17} \mathrm{O}_{2}:$ 205.1229. Found: 205.1235.

1-Methyl-4-phenyl-2,6-dioxabicyclo[2.2.1]heptane 2c

${ }^{1} \mathrm{H}-\mathrm{NMR}\left(300 \mathrm{MHz}, \mathrm{CDCl}_{3}\right)$ : $\delta 1.67$ (s, 3H), $2.20(\mathrm{~s}, 2 \mathrm{H}), 4.10(\mathrm{~m}, 4 \mathrm{H}), 7.15-7.40(\mathrm{~m}$,<smiles>CC12OCC(c3ccccc3)(CO1)CO2</smiles>
5H). ${ }^{13} \mathrm{C}-\mathrm{NMR}\left(75 \mathrm{MHz}, \mathrm{CDCl}_{3}\right): \delta 18.0,44.4,52.8,77.6,109.1,126.4,127.6,128.8$, 136.4. CI-MS $\left(\mathrm{NH}_{3}\right) \mathrm{m} / \mathrm{z} 208\left[\mathrm{M}+\mathrm{NH}_{4}\right]^{+}, 191[\mathrm{M}+\mathrm{H}]^{+}$. DCI/ $\mathrm{NH}_{3}-\mathrm{HRMS}$ Calcd for $\mathrm{C}_{12} \mathrm{H}_{15} \mathrm{O}_{2}$ : 191.1072. Found: 191.1075.

4-n-Butyl-1-methyl-2,6-dioxabicyclo[2.2.1]heptane 2d

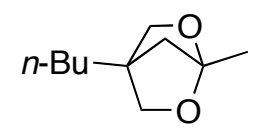

${ }^{1} \mathrm{H}-\mathrm{NMR}\left(400 \mathrm{MHz}, \mathrm{CDCl}_{3}\right): \delta 0.88(\mathrm{t}, 3 \mathrm{H}, J=6.7 \mathrm{~Hz}), 1.21-1.58(\mathrm{~m}, 9 \mathrm{H}), 1.66(\mathrm{~s}$, $2 \mathrm{H}), 3.71-3.78(\mathrm{~m}, 4 \mathrm{H}) .{ }^{13} \mathrm{C}-\mathrm{NMR}\left(75 \mathrm{MHz}, \mathrm{C}_{6} \mathrm{D}_{6}\right): \delta 13.8,18.3,23.5,27.9,28.2$, 44.9, 49.0, 76.2, 108.3. CI-MS $\left(\mathrm{NH}_{3}\right) \mathrm{m} / \mathrm{z} 188\left[\mathrm{M}+\mathrm{NH}_{4}\right]^{+}, 171[\mathrm{M}+\mathrm{H}]^{+} . \mathrm{DCI} / \mathrm{NH}_{3}{ }^{-}$ HRMS Calculated for $\mathrm{C}_{10} \mathrm{H}_{19} \mathrm{O}_{2}: 171.1385$. Found: 171.1387. 
1-Methyl-4-[3'-phenylprop-2'-enyl]-2,6-dioxabicyclo[2.2.2]octane 2e

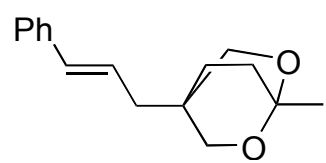

${ }^{1} \mathrm{H}-\mathrm{NMR}\left(400 \mathrm{MHz}, \mathrm{CDCl}_{3}\right): \delta 1.20(\mathrm{~s}, 3 \mathrm{H}), 1.63-1.97(\mathrm{~m}, 6 \mathrm{H}), 3.72-3.82(\mathrm{~m}$, $4 \mathrm{H}), 5.96(\mathrm{dt}, 1 \mathrm{H}, J=15.6,7.5 \mathrm{~Hz}), 6.28(\mathrm{~d}, 1 \mathrm{H}, J=15.6 \mathrm{~Hz}), 7.13-7.27(\mathrm{~m}$, $5 \mathrm{H}) .{ }^{13} \mathrm{C}-\mathrm{NMR}\left(75 \mathrm{MHz}, \mathrm{CDCl}_{3}\right): \delta 25.0,28.0,32.0,32.8,37.4,76.8,94.7$, 123.8, 126.1, 127.4, 128.6, 133.3, 137.0. CI-MS $\left(\mathrm{NH}_{3}\right) \mathrm{m} / \mathrm{z} 262\left[\mathrm{M}+\mathrm{NH}_{4}\right]^{+}$, $245[\mathrm{M}+\mathrm{H}]^{+}$. DCI/ $\mathrm{NH}_{3}-\mathrm{HRMS}$ Calculated for $\mathrm{C}_{16} \mathrm{H}_{21} \mathrm{O}_{2}$ : 245.1542. Found: 245.1547 .

1-Methyl-4-[prop-2'-enyl]-2,6-dioxabicyclo[2.2.2]octane $\mathbf{2 f}$

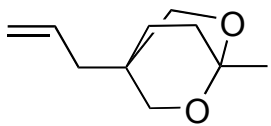

${ }^{1} \mathrm{H}-\mathrm{NMR}\left(400 \mathrm{MHz}, \mathrm{CDCl}_{3}\right)$ : $\delta 1.25(\mathrm{~s}, 3 \mathrm{H}), 1.63-1.97(\mathrm{~m}, 6 \mathrm{H}), 3.72-3.82(\mathrm{~m}$, $4 \mathrm{H}), 5.00(\mathrm{dd}, 1 \mathrm{H}, J=16.8,1.7 \mathrm{~Hz}), 5.04(\mathrm{dd}, 1 \mathrm{H}, J=8.9,1.7 \mathrm{~Hz}), 5.63(\mathrm{ddt}$, $1 \mathrm{H}, J=16.8,8.9,7.5 \mathrm{~Hz}) .{ }^{13} \mathrm{C}-\mathrm{NMR}\left(75 \mathrm{MHz}, \mathrm{CDCl}_{3}\right): \delta 25.0,27.9,31.9$, 32.0, 38.3, 72.7, 94.6, 118.2, 132.2. CI-MS $\left(\mathrm{NH}_{3}\right) \mathrm{m} / \mathrm{z} 169[\mathrm{M}+\mathrm{H}]^{+}$. DCI/ $\mathrm{NH}_{3}{ }^{-}$ HRMS Calculated for $\mathrm{C}_{10} \mathrm{H}_{17} \mathrm{O}_{2}: 169.1150$. Found: 169.1231 .

1-Methyl-4-[prop-2'-enyl]-2,6-dioxabicyclo[2.2.1]heptane 2g

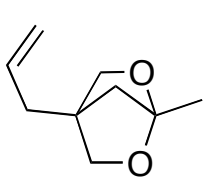

${ }^{1} \mathrm{H}-\mathrm{NMR}\left(300 \mathrm{MHz}, \mathrm{CDCl}_{3}\right): \delta 1.52(\mathrm{~s}, 3 \mathrm{H}), 1.68(\mathrm{~s}, 2 \mathrm{H}), 2.32(\mathrm{~d}, J=7.4 \mathrm{~Hz}, 2 \mathrm{H})$, $3.74(\mathrm{~m}, 4 \mathrm{H}), 4.95-5.15(\mathrm{~m}, 2 \mathrm{H}), 5.60-5.85(\mathrm{~m}, 1 \mathrm{H}) .{ }^{13} \mathrm{C}-\mathrm{NMR}\left(75 \mathrm{MHz}, \mathrm{CDCl}_{3}\right): \delta$ 17.9, 32.8, 44.2, 48.7, 75.8, 108.4, 117.8, 133.3. CI-MS $\left(\mathrm{NH}_{3}\right) \mathrm{m} / \mathrm{z} 172\left[\mathrm{M}+\mathrm{NH}_{4}\right]^{+}, 155$ $[\mathrm{M}+\mathrm{H}]^{+}$. DCI/NH $\mathrm{NH}_{3}-\mathrm{HRMS}$ Calcd for $\mathrm{C}_{9} \mathrm{H}_{15} \mathrm{O}_{2}: 155.1072$. Found: 155.1069 .

1-Methyl-4-[cyclohex-2'-enyl]-2,6-dioxabicyclo[2.2.1]heptane $\mathbf{2 h}$

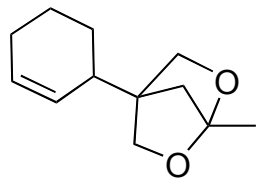

${ }^{1} \mathrm{H}-\mathrm{NMR}\left(400 \mathrm{MHz}, \mathrm{CDCl}_{3}\right): \delta$ 1.29-1.98 $(\mathrm{m}, 11 \mathrm{H}), 2.40-2.50(\mathrm{~m}, 1 \mathrm{H}), 3.74-$ $3.85(\mathrm{~m}, 4 \mathrm{H}), 5.51(\mathrm{~d}, 1 \mathrm{H}, J=10.2 \mathrm{~Hz}), 5.73-5.78(\mathrm{~m}, 1 \mathrm{H}) .{ }^{13} \mathrm{C}-\mathrm{NMR}(75$ $\left.\mathrm{MHz}, \mathrm{CDCl}_{3}\right): \delta 17.9,, 21.9,24.9,25.8,35.3,43.0,52.5,74.5,75.3,108.0$, 127.0, 129.3. CI-MS $\left(\mathrm{NH}_{3}\right) \mathrm{m} / \mathrm{z} 195[\mathrm{M}+\mathrm{H}]^{+}$. DCI/ $\mathrm{NH}_{3}-\mathrm{HRMS}$ Calculated for $\mathrm{C}_{12} \mathrm{H}_{19} \mathrm{O}_{2}$ : 195.1385. Found: 195.1390.

1-Methyl-4-[3'-methyl-but-2'-enyl]-2,6-dioxabicyclo[2.2.1]heptane 2i<smiles>CC(C)=CCC12COC(C)(OC1)O2</smiles>

${ }^{1} \mathrm{H}-\mathrm{NMR}\left(400 \mathrm{MHz}, \mathrm{CDCl}_{3}\right): \delta 1.51(\mathrm{~s}, 3 \mathrm{H}), 1.58(\mathrm{~s}, 3 \mathrm{H}), 1.67(\mathrm{~s}, 2 \mathrm{H}), 1.69(\mathrm{~s}$, $3 \mathrm{H}), 2.23(\mathrm{~d}, 2 \mathrm{H}, J=7.6 \mathrm{~Hz}), 3.70-3.76(\mathrm{~m}, 4 \mathrm{H}), 5.06-5.12(\mathrm{~m}, 1 \mathrm{H}) .{ }^{13} \mathrm{C}-\mathrm{NMR}$ $\left(75 \mathrm{MHz}, \mathrm{CDCl}_{3}\right): \delta 17.6,17.9,25.7,26.5,44.2,49.6,76.3,108.4,118.9$, 134.4. CI-MS $\left(\mathrm{NH}_{3}\right) \mathrm{m} / \mathrm{z} 183[\mathrm{M}+\mathrm{H}]^{+}$. DCI/ $\mathrm{NH}_{3}-\mathrm{HRMS}$ Calculated for $\mathrm{C}_{11} \mathrm{H}_{19} \mathrm{O}_{2}: 183.1385$. Found: 183.1379 .

3-hydroxymethyl-3-prop-2'-ynylpentane-1,4-diol 1j (2 diastereomers)

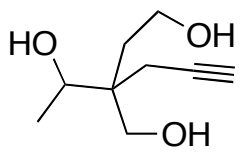

${ }^{1} \mathrm{H}-\mathrm{NMR}\left(300 \mathrm{MHz}, \mathrm{CDCl}_{3}\right) \delta$ 1.19-1,23 (m, 3H), 1.50-1.70 (m, 2H), $2.00(\mathrm{~m}$, $1 \mathrm{H}), 2.10-2.40(\mathrm{~m}, 2 \mathrm{H}), 3.40-4.20 .(\mathrm{m}, 5 \mathrm{H}) .{ }^{13} \mathrm{C}-\mathrm{NMR}\left(75 \mathrm{MHz}, \mathrm{CDCl}_{3}\right) \delta$ 16.6, 16.7, 20.7, 22.1, 28.7, 32.8, 33.5, 42.4, 42.7, 57.1, 57.2, 65.1, 65.6, 69.8, 70.2, 70.7, 80.0, 80.2. CI-MS $\left(\mathrm{NH}_{3}\right) \mathrm{m} / \mathrm{z} 190\left[\mathrm{M}+\mathrm{NH}_{4}\right]^{+}, 173[\mathrm{M}+\mathrm{H}]^{+}$. DCI/ $\mathrm{NH}_{3}-\mathrm{HRMS}$ Calcd for $\mathrm{C}_{9} \mathrm{H}_{17} \mathrm{O}_{3}: 173.1178$. Found: 173.1174 . 
4-[1'-hydroxyethyl]-1-methyl-2,7-dioxabicyclo[3.2.1]octane $\mathbf{2} \mathbf{j}$

(4 diastereomers)

${ }^{1} \mathrm{H}-\mathrm{NMR}\left(300 \mathrm{MHz}, \mathrm{CDCl}_{3}\right) \delta$ 1.10-1.30 (m, 3H), $1.40(\mathrm{~m}, 3 \mathrm{H}), 1.50-1.80(\mathrm{~m}$,<smiles>CC(O)C12CCOC(C)(CO1)C2</smiles>
$4 \mathrm{H}), 2.20(\mathrm{~s}, 1 \mathrm{H}), 3.40-4.30(\mathrm{~m}, 5 \mathrm{H}) .{ }^{13} \mathrm{C}-\mathrm{NMR}\left(75 \mathrm{MHz}, \mathrm{CDCl}_{3}\right): \delta 13.7$, 17.7, 19.0, 19.6, 23.8, 24.2, 24.5, 27.5, 28.5, 29.3, 33.1, 42.2, 44.6, 44.7, 45.5, $46.4,46.6,48.3,48.5,59.8,60.2,60.4,65.6,66.0,70.7,71.5,73.9,75.9,79.6$, 80.5, 104.3, 104.9, 106.3, 106.5. CI-MS $\left(\mathrm{NH}_{3}\right) \mathrm{m} / \mathrm{z} 190\left[\mathrm{M}+\mathrm{NH}_{4}\right]^{+}, 173$ $[\mathrm{M}+\mathrm{H}]^{+}$. DCI/NH $\mathrm{NH}_{3}-\mathrm{HRMS}$ Calculated for DCI/ $\mathrm{NH}_{3}-\mathrm{HRMS}$ Calcd for $\mathrm{C}_{9} \mathrm{H}_{17} \mathrm{O}_{3}$ : 173.1178. Found: 173.1175. 